\title{
Towards an Effective Importance Sampling in Monte Carlo Simulations of a System with a Complex Action
}

\author{
Konstantinos N. Anagnostopoulos* ${ }^{\dagger}$ \\ Physics Department, National Technical University of Athens, Zografou Campus, 157-80 \\ Zografou, Greece \\ E-mail: konstantemail.ntua.qr
}

\section{Takehiro Azuma}

Institute for Fundamental Sciences, Setsunan University, 17-8 Ikeda Nakamachi, Neyagawa, Osaka 572-8508, Japan

E-mail: azumadmpq.setsunan.ac.jp

\section{Jun Nishimura}

High Energy Accelerator Research Organization (KEK) and Graduate University for Advanced Studies (SOKENDAI), 1-1 Oho, Tsukuba 305-0801, Japan

E-mail: jnishiepost.kek.jp

\begin{abstract}
The sign problem is a notorious problem, which occurs in Monte Carlo simulations of a system with a partition function whose integrand is not positive. One way to simulate such a system is to use the factorization method where one enforces sampling in the part of the configuration space which gives important contribution to the partition function. This is accomplished by using constraints on some observables chosen appropriately and minimizing the free energy associated with their joint distribution functions. These observables are maximally correlated with the complex phase. Observables not in this set essentially decouple from the phase and can be calculated without the sign problem in the corresponding "microcanonical" ensemble. These ideas are applied on a simple matrix model with very strong sign problem and the results are found to be consistent with analytic calculations using the Gaussian Expansion Method.
\end{abstract}

The XXIX International Symposium on Lattice Field Theory - Lattice 2011

July 10-16, 2011

Squaw Valley, Lake Tahoe, California

\footnotetext{
* Speaker.

$\dagger$ The work of K.N.A. was partially funded by the National Technical University of Athens through the Basic Research Support Programmes 2009 and 2010. The work of T.A. and J.N. is supported in part by Grant-in-Aid for Scientific Research (No. 23740211 for T.A. and 19340066, 20540286 for J.N.) from Japan Society for the Promotion of Science.
} 


\section{Introduction}

Monte Carlo simulation is an essential tool in the non perturbative study of a quantum field theory and has provided a wealth of information and insight in high energy physics. Its success lies in the fact that algorithmic importance sampling of a very small fraction of configurations gives a very accurate estimate of expectation values of physical observables. By stochastically constructing Markov chains of configurations, the system quickly thermalizes and one is able to sample the extremely small subset $\mathscr{R}$ of the configuration space $\mathscr{C}$ that gives the important contribution to the partition function. $\mathscr{R}$ is determined by the competition of entropy with the Boltzmann factor of the action. For an observable $\mathscr{O}$ whose value on the configuration $\mu$ is $\mathscr{O}[\mu]$, the Markov chain sample $\left\{\mu_{1}, \mu_{2}, \ldots, \mu_{M}\right\}$ is constructed with sampling probability $P_{\mu}$ and $\langle\mathscr{O}\rangle$ 's estimator is

$$
\overline{\mathscr{O}}=\frac{\sum_{i=1}^{M}\left(P_{\mu_{i}}\right)^{-1} \mathscr{O}\left[\mu_{i}\right] \mathrm{e}^{-S\left[\mu_{i}\right]}}{\sum_{i=1}^{M}\left(P_{\mu_{i}}\right)^{-1} \mathrm{e}^{-S\left[\mu_{i}\right]}} .
$$

By choosing $P_{\mu} \propto \mathrm{e}^{-S\left[\mu_{i}\right]}$, the above sum is easily performed numerically without terms whose size varies exponentially with the system size ${ }^{1}$.

This approach breaks down in the case where the action of the system is complex and almost all configurations contribute a non positive term in the partition function. The sample is constructed by simulating a phase quenched model. One then mostly samples a subset $\mathscr{R}_{0}$ of $\mathscr{C}$ whose overlap with $\mathscr{R}$ is exponentially small with system size. The reason is that besides entropy and the Boltzmann factor of the real part of the action, the fluctuations of the imaginary part of the action become a determining factor in the suppression of configurations. Moreover, if one takes a simple reweighting approach to estimating $\langle\mathscr{O}\rangle$, one obtains

$$
\overline{\mathscr{O}}=\frac{\sum_{i=1}^{M} \mathscr{O}\left[\mu_{i}\right] \mathrm{e}^{i \Gamma\left[\mu_{i}\right]}}{\sum_{i=1}^{M} \mathrm{e}^{i \Gamma\left[\mu_{i}\right]}}
$$

where $\Gamma[\mu]$ is the imaginary part of the value of the action on $\mu$. Then, besides the small overlap, one has to sum terms that oscillate wildly due to the exponential that appears in the numerator and the denominator. The oscillations are much stronger away from the stationary configurations of $\Gamma$ which is usually what happens when we sample in $\mathscr{R}_{0}$.

An approach to studying such systems is the factorization method, originally proposed in [四], used in [[] and recently generalized in [B]. The key point is the selection of a maximal set of observables

$$
\Sigma=\left\{\mathscr{O}_{k} \mid k=1, \ldots, n\right\}
$$

which are strongly correlated with the phase $\mathrm{e}^{i \Gamma}$. The idea is that by solving saddle point equations for the minimum of the "free energy" associated with the distribution functions of these observables, one can essentially determine the region $\mathscr{R}$. The solutions are obtained by performing Monte Carlo simulations on selected subspaces of $\mathscr{C}$ by constraining the values of these observables. Further calculations of any other observable $\mathscr{O}$ can be done by essentially sampling in $\mathscr{R}$ and their

\footnotetext{
${ }^{1}$ like e.g. in multihistogramming.
} 


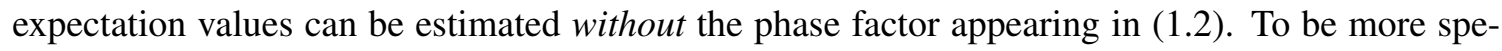
cific, if $\left\{\bar{x}_{1}, \bar{x}_{2}, \ldots, \bar{x}_{n}\right\}$ are the values ${ }^{2}$ of $\left\{\mathscr{O}_{1}, \mathscr{O}_{2}, \ldots, \mathscr{O}_{n}\right\}$ that are the solutions to the free energy minimization equations, then one finds that

$$
\langle\mathscr{O}\rangle \approx\langle\mathscr{O}\rangle_{\bar{x}_{1}, \bar{x}_{2}, \ldots, \bar{x}_{n}}
$$

where $\langle\ldots\rangle_{\bar{x}_{1}, \bar{x}_{2}, \ldots, \bar{x}_{n}}$ are expectation values in a "microcanonical" system where $\mathscr{O}_{k}$ are constrained to be equal to $\bar{x}_{k}$. The right hand side of (때) has no complex action problem. The complex action problem has been reduced to computing the solution $\left\{\bar{x}_{1}, \ldots, \bar{x}_{n}\right\}$ and this is greatly improved by factorizing the phase factor and taking advantage of its (hopefully) nice scaling properties.

In this talk, the above general statements will be made concrete in a specific example. A pedestrian's approach is adopted for presenting the main ideas and the reader is referred to [B]] for the details. A simple matrix model with very strong complex action problem is studied and is shown how to compute the set $\Sigma$ and the solution $\left\{\bar{x}_{1}, \ldots, \bar{x}_{n}\right\}$. Solving the equations that give the stationary points of $\Gamma$ plays an important role in determining $\Sigma$. The nice scaling properties of the distribution functions are heavily used in the computation of the solution $\left\{\bar{x}_{1}, \ldots, \bar{x}_{n}\right\}$.

Since the method can in principle be applied to any system with a complex action problem, we hope that our exposition will contribute to a successful application of the method to other interesting problems in lattice field theory and elsewhere.

\section{The Model}

Matrix models have been studied extensively in the context of non perturbative formulations of string theory and in the study of gauge/gravity duality. Monte Carlo simulations have contributed crucially in the study of the large $N$ limit of supersymmetric matrix models [团], in providing first principle evidence of gauge/gravity duality and in explaining the thermodynamics of certain black hole solutions in terms of microscopic string degrees of freedom [[]].

The large $N$ limit of the IIB matrix model [ [6] has been proposed as a non perturbative definition of string theory. In this model, space-time emerges as the eigenvalue distribution of the bosonic matrices which makes possible to study the scenario of dynamical compactification of extra dimensions. This happens via spontaneous symmetry breaking of the rotational symmetry (SSB) of the model. Calculations using the Gaussian Expansion Method (GEM) support the realization of such a scenario [ []]. Monte Carlo simulations can play an important role in confirming those results from first principle calculations and in elucidating the mechanism that is responsible for SSB. The strong fluctuations of the phase $\mathrm{e}^{i \Gamma}$ favour length scales over which spacetime extends which are quite different than in the phase quenched model [四]. A simple matrix model that realizes the above scenario has been proposed in []]. It is defined by the partition function

$$
Z=\int d A d \psi d \bar{\psi} \mathrm{e}^{-\left(S_{\mathrm{b}}+S_{\mathrm{f}}\right)} \quad \text { where } \quad S_{\mathrm{b}}=\frac{1}{2} N \operatorname{tr}\left(A_{\mu}\right)^{2}, \quad S_{\mathrm{f}}=-\bar{\psi}_{\alpha}^{f}\left(\Gamma_{\mu}\right)_{\alpha \beta} A_{\mu} \psi_{\beta}^{f} .
$$

$A_{\mu}(\mu=1,2,3,4)$ are $N \times N$ hermitian matrices, and $\bar{\psi}_{\alpha}^{f}$ and $\psi_{\alpha}^{f}\left(\alpha=1,2, f=1, \ldots, N_{f}\right)$ are $N$-dimensional row and column vectors. The actions (2. $($ ) have an $\mathrm{SO}(4)$ symmetry, where the

\footnotetext{
${ }^{2}$ More precisely $\bar{x}_{k}$ are equal to the values of $\mathscr{O}_{k} /\left\langle\mathscr{O}_{k}\right\rangle_{0}$ as it will be explained in the next paragraph.
} 
bosonic variables $A_{\mu}$ transform as vectors and the fermionic variables transform as Weyl spinors. Integrating out the fermions, we obtain $Z=\int d A \mathrm{e}^{-S_{\mathrm{b}}} Z_{\mathrm{f}}[A]$, where $Z_{\mathrm{f}}[A]=(\operatorname{det} \mathscr{D})^{N_{\mathrm{f}}}$ and $\mathscr{D}=\Gamma_{\mu} A_{\mu}$ is a $2 N \times 2 N$ matrix. The fermion determinant $\operatorname{det} \mathscr{D}$ for a single flavor is complex in general but it turns out to be real for configurations with $A_{4}=0$ and that the phase of the determinant becomes stationary for configurations with $A_{4}=A_{3}=0$ [ए]]. We take the large- $N$ limit with $r=N_{\mathrm{f}} / N$ fixed, which corresponds to the Veneziano limit.

The order parameters of SSB of $\mathrm{SO}(4)$ are the expectation values of the eigenvalues of the "moment of inertia tensor" $T_{\mu \nu}=\frac{1}{N} \operatorname{tr}\left(A_{\mu} A_{v}\right)$. These are ordered as $\lambda_{1}>\lambda_{2}>\lambda_{3}>\lambda_{4}>0$ and if their VEVs turn out to be unequal in the large- $N$ limit, it signals the SSB of SO(4). GEM calculations [Q] show that $\mathrm{SO}(4)$ breaks down to $\mathrm{SO}(2)$ for all $r>0$ and Monte Carlo simulations are consistent with this result [B]].

The "phase quenched model" is defined by

$$
Z_{0}=\int d A e^{-S_{0}[A]}, \quad S_{0}[A]=S_{\mathrm{b}}[A]-N_{\mathrm{f}} \log |\operatorname{det} \mathscr{D}[A]| .
$$

and in this case SSB is absent. In fact $\left\langle\lambda_{n}\right\rangle_{0}=1+\frac{r}{2}$ for all $n=1,2,3,4$ where the VEVs $\langle\cdot\rangle_{0}$ are taken with respect to ( $(2.2)$. In the following, we will study the eigenvalues of $T_{\mu \nu}$ normalized to their phase quenched expectation values and we denote $\tilde{\lambda}_{n}=\lambda_{n} /\left\langle\lambda_{n}\right\rangle_{0}$. Deviation of $\left\langle\tilde{\lambda}_{n}\right\rangle$ from 1 indicates a strong effect of the complex phase. In order to simulate (2. $\int d A \mathrm{e}^{-S_{0}[A]} \mathrm{e}^{i \Gamma[A]}$, where due to nonzero $\Gamma[A]$ for generic configurations, the system turns out to have a very strong complex action problem. Due to the stationarity of the phase for lower dimensional configurations, the eigenvalues $\tilde{\lambda}_{n}$ are chosen for the application of the factorization method.

We study the distribution function $\rho\left(x_{1}, x_{2}, x_{3}, x_{4}\right)=\left\langle\prod_{k=1}^{4} \delta\left(x_{k}-\tilde{\lambda}_{k}\right)\right\rangle$ and the corresponding one in the phase quenched model $\rho^{(0)}\left(x_{1}, x_{2}, x_{3}, x_{4}\right)=\left\langle\prod_{k=1}^{4} \delta\left(x_{k}-\tilde{\lambda}_{k}\right)\right\rangle_{0}$. By defining the "microcanonical ensemble" $Z_{x_{1}, x_{2}, x_{3}, x_{4}}=\int d A \mathrm{e}^{-S_{0}} \prod_{k=1}^{4} \delta\left(x_{k}-\tilde{\lambda}_{k}\right)$, we define the function $w\left(x_{1}, x_{2}, x_{3}, x_{4}\right)=$ $\left\langle\mathrm{e}^{i \Gamma}\right\rangle_{x_{1}, x_{2}, x_{3}, x_{4}}$ and one finds that

$$
\rho\left(x_{1}, x_{2}, x_{3}, x_{4}\right)=\frac{1}{C} \rho^{(0)}\left(x_{1}, x_{2}, x_{3}, x_{4}\right) w\left(x_{1}, x_{2}, x_{3}, x_{4}\right),
$$

where $C=\left\langle\mathrm{e}^{i \Gamma}\right\rangle_{0}$. The minimum of $\mathscr{F}\left(x_{1}, x_{2}, x_{3}, x_{4}\right)=-\ln \rho\left(x_{1}, x_{2}, x_{3}, x_{4}\right)$ is the estimator for $\left\{\left\langle\tilde{\lambda}_{1}\right\rangle,\left\langle\tilde{\lambda}_{2}\right\rangle,\left\langle\tilde{\lambda}_{3}\right\rangle,\left\langle\tilde{\lambda}_{4}\right\rangle\right\}$. One has to solve the saddle point equations

$$
\frac{1}{N^{2}} f_{n}^{(0)}\left(x_{1}, x_{2}, x_{3}, x_{4}\right)=-\frac{\partial}{\partial x_{n}} \Phi\left(x_{1}, x_{2}, x_{3}, x_{4}\right), \quad n=1,2,3,4
$$

where $f_{n}^{(0)}\left(x_{1}, x_{2}, x_{3}, x_{4}\right)=\frac{\partial}{\partial x_{n}} \ln \rho^{(0)}\left(x_{1}, x_{2}, x_{3}, x_{4}\right), \Phi\left(x_{1}, x_{2}, x_{3}, x_{4}\right)=\lim _{N \rightarrow \infty} \frac{1}{N^{2}} \ln w\left(x_{1}, x_{2}, x_{3}, x_{4}\right)$. The nice scaling properties of $f_{n}^{(0)}\left(x_{1}, x_{2}, x_{3}, x_{4}\right)$ and $\Phi\left(x_{1}, x_{2}, x_{3}, x_{4}\right)$ allow for extrapolations in $N$ and $x_{n}$. It is also important to note that the error in $\left\langle\tilde{\lambda}_{n}\right\rangle$ does not propagate exponentially in $N$.

Looking for the full solution of (2.4) using Monte Carlo is a formidable task. Therefore, guided by the GEM results in [Q] we look for $\mathrm{SO}(3)$ and $\mathrm{SO}(2)$ symmetric solutions. For lack of space we discuss the $\mathrm{SO}(3)$ symmetric vacuum and the interested reader is referred to [目] for the $\mathrm{SO}(2)$ case. We take $x_{1}=x_{2}=x_{3}>1>x_{4}$ and define the reduced functions $\rho_{\mathrm{SO}(3)}^{(0)}(x, y)=\rho^{(0)}(x, x, x, y)$, $w_{\mathrm{SO}(3)}(x, y)=w(x, x, x, y), f_{\mathrm{SO}(3), x_{i}}^{(0)}(x, y)=\frac{\partial}{\partial x_{i}} \ln \rho_{\mathrm{SO}(3)}^{(0)}(x, y)$ and $\Phi_{\mathrm{SO}(3)}(x, y)=\lim _{N \rightarrow \infty} \frac{1}{N^{2}} \ln w_{\mathrm{SO}(3)}(x, y)$. 

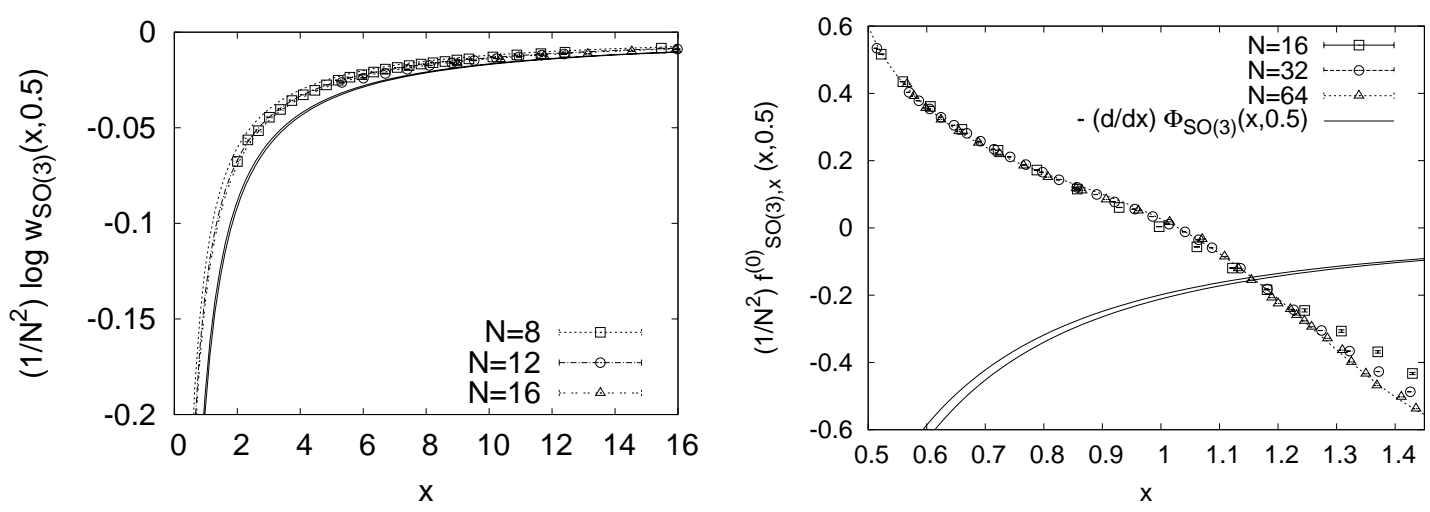

Figure 1: (Left) The function $\frac{1}{N^{2}} \log w_{\mathrm{SO}(3)}(x, 0.5)$ is plotted against $x$ for $N=8,12,16$. The solid lines represent the function $\Phi_{\mathrm{SO}(3)}(x, 0.5)$ obtained by extrapolation to $N=\infty$ as described in [B]]. (Right) The function $\frac{1}{N^{2}} f_{\mathrm{SO}(3), x}^{(0)}(x, 0.5)$ is plotted against $x$ for $N=16,32,64$. The solid lines represent $-\frac{\partial}{\partial x} \Phi_{\mathrm{SO}(3)}(x, 0.5)$ obtained from the plot on the left.

\begin{tabular}{|c|c|c|c|c|}
\hline ansatz & \multicolumn{2}{|c|}{$\mathrm{SO}(3)$} & \multicolumn{2}{c|}{$\mathrm{SO}(2)$} \\
\hline method & factorization & GEM & factorization & GEM \\
\hline$\left\langle\tilde{\lambda}_{1}\right\rangle$ & - & 1.17 & - & 1.4 \\
$\left\langle\tilde{\lambda}_{2}\right\rangle$ & - & 1.17 & $1.373(2)$ & 1.4 \\
$\left\langle\tilde{\lambda}_{3}\right\rangle$ & $1.151(2)$ & 1.17 & $0.649(4)$ & 0.7 \\
$\left\langle\tilde{\lambda}_{4}\right\rangle$ & $0.59(2)$ & 0.5 & $0.551(2)$ & 0.5 \\
\hline
\end{tabular}

Table 1: The results for the normalized eigenvalues $\left\langle\tilde{\lambda}_{n}\right\rangle$ for $r=1$ obtained by the factorization method for the $\mathrm{SO}(3)$ and $\mathrm{SO}(2)$ symmetric vacua. The dash implies that the result should be the same as the one below in the same column due to the imposed symmetry. We also show the GEM results obtained at $N=\infty$ in ref. [Q].

Equations (R.4) become

$$
\frac{1}{N^{2}} f_{\mathrm{SO}(3), x}^{(0)}(x, y)=-\frac{\partial}{\partial x} \Phi_{\mathrm{SO}(3)}(x, y), \quad \frac{1}{N^{2}} f_{\mathrm{SO}(3), y}^{(0)}(x, y)=-\frac{\partial}{\partial y} \Phi_{\mathrm{SO}(3)}(x, y) .
$$

In the following we fix $r=N_{f} / N=1$ and from the GEM results [ [Q] we expect that the $\mathrm{SO}$ (3) symmetric solution is $\left(x_{\mathrm{GEM}}, y_{\mathrm{GEM}}\right) \approx(1.17,0.50)$. We first fix $y=y_{\mathrm{GEM}}=0.50$ and solve for $x$. The

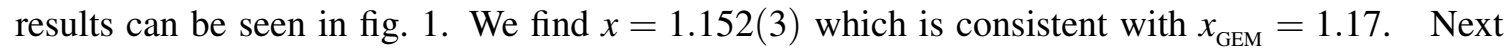
we fix $x=x_{\text {GEM }}=1.17$ and solve for $y$. We repeat the same procedure for the $\mathrm{SO}(2)$ symmetric vacuum and our results are summarized in Table $\mathbb{\text { I. }}$.

Including more variables in the analysis is straightforward. If $(X, X, Y, Z)$ is the absolute maximum of $\rho\left(x_{1}, x_{2}, x_{3}, x_{4}\right)$, consider the "microcanonical ensemble" $\langle\cdot\rangle_{X, X, Y, Z}$ and for an operator $\mathscr{O}$ define $\rho_{\mathscr{O}}(x)=\frac{\left\langle\delta(x-\widetilde{\mathscr{O}}) \mathrm{e}^{\mathrm{i} \Gamma}\right\rangle_{X, X, Y, Z}}{\left\langle\mathrm{e}^{\Gamma}\right\rangle_{X, X, Y, Z}}, \rho_{\mathscr{O}}^{(0)}(x)=\langle\delta(x-\widetilde{\mathscr{O}})\rangle_{X, X, Y, Z}$ where $\widetilde{\mathscr{O}}=\mathscr{O} /\langle\mathscr{O}\rangle_{0}$. Then $\rho_{\mathscr{O}}(x)=\frac{1}{C^{\prime}} \rho_{\mathscr{O}}^{(0)}(x) w_{\mathscr{O}}(x)$ where $C^{\prime}=\left\langle\mathrm{e}^{i \Gamma}\right\rangle_{X, X, Y, Z}$ and $w_{\mathscr{O}}(x)=\frac{\left\langle\delta(x-\widetilde{\mathscr{O}}) \mathrm{e}^{i \Gamma}\right\rangle_{X, X, Y, Z}}{\langle\delta(x-\widetilde{\mathscr{O}})\rangle_{X, X, Y, Z}}$. Next we determine $\langle\mathscr{O}\rangle$ from $\frac{1}{N^{2}} f_{\mathscr{O}}^{(0)}=-\frac{d}{d x} \Phi_{\mathscr{O}}(x)$, where $f_{\mathscr{O}}^{(0)}=\frac{d}{d x} \ln \rho_{\mathscr{O}}^{(0)}(x)$ and $\Phi_{\mathscr{O}}(x)=\lim _{N \rightarrow \infty} \frac{1}{N^{2}} \ln w_{\mathscr{O}}(x)$. If $\langle\mathscr{O}\rangle$ does not shift much from $\langle\mathscr{O}\rangle_{X, X, Y, Z}$ then the overlap problem for $\mathscr{O}$ is not severe. Then one can 

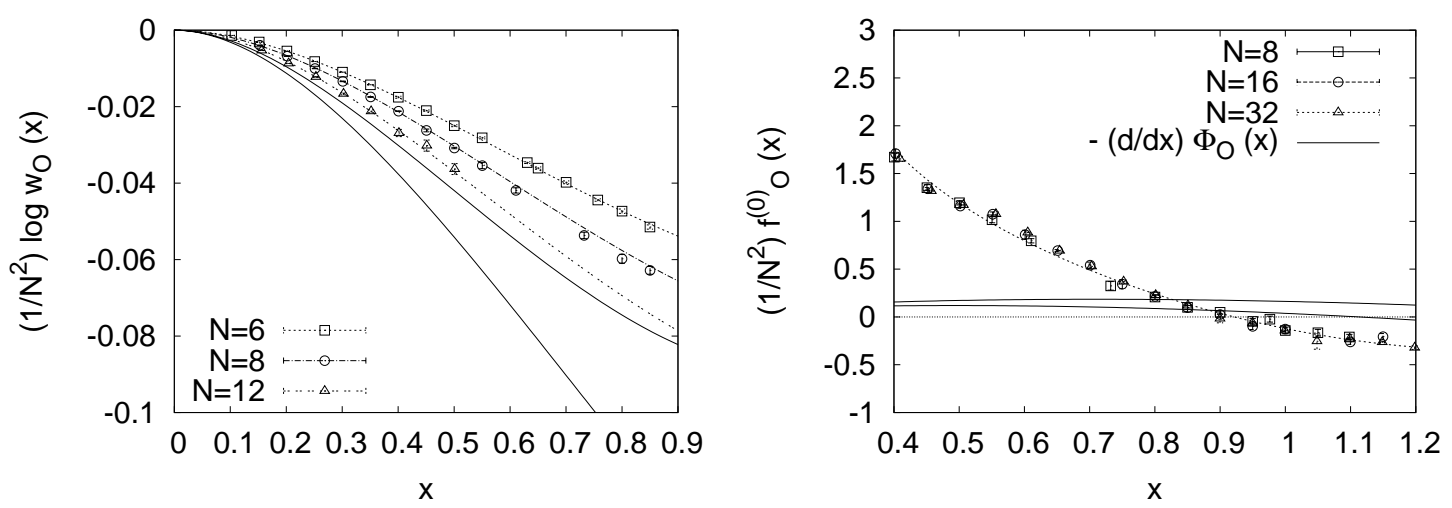

Figure 2: (Left) The function $\frac{1}{N^{2}} \log w_{\mathscr{O}}(x)$ is plotted against $x$ for $N=6,8,12$. We also plot the function $\Phi_{\mathscr{O}}(x)$ obtained by extrapolation to $N=\infty$ as described in [[]]. The two solid lines represent the margin of error. (Right) The function $\frac{1}{N^{2}} \frac{d}{d x} \log \rho_{\mathscr{O}}^{(0)}(x)$ is plotted for $N=8,16,32$. We also plot $-\frac{d}{d x} \Phi_{\mathscr{O}}(x)$ obtained from the plot on the left.

show that [3]

$$
\langle\mathscr{O}\rangle_{X, X, Y, Z} \approx \frac{\left\langle\mathscr{O} \mathrm{e}^{i \Gamma}\right\rangle_{X, X, Y, Z}}{\left\langle\mathrm{e}^{i \Gamma}\right\rangle_{X, X, Y, Z}}
$$

i.e. correlation of $\mathscr{O}$ and $\mathrm{e}^{i \Gamma}$ within $\langle\cdot\rangle_{X, X, Y, Z}$ is small. The advantage of this relation is that the phase factors out and one can calculate the expectation value of $\mathscr{O}$ within the microcanonical ensemble without the sign problem.

We test the above relations by considering the observable $\mathscr{O}=-\frac{1}{N} \sum_{\mu \neq \nu} \operatorname{tr}\left[A_{\mu}, A_{v}\right]^{2}$. As $x \ll 1$ the dominant configurations are approximately simultaneously diagonalizable $\left[A_{\mu}, A_{v}\right] \approx 0$ and $\operatorname{det} \mathscr{D} \geq 0$. Therefore $\mathscr{O}$ can potentially have strong correlations with the phase factor. From fig. $\square$ (Left) we find that $\frac{1}{N^{2}} \log w_{\mathscr{O}}(x)$ approaches zero for $x \rightarrow 0$ as expected. From fig. $\square$ (Right) we find that the effect of the phase is to shift the estimate of $\langle\widetilde{\mathscr{O}}\rangle$ by $\Delta x=0.07(3)$. On the other hand, the standard deviation of the distribution $\rho_{\mathscr{O}}^{(0)}(x)$ is estimated as $\sigma \sim 0.7 / N$ from the slope of the function plotted in fig. $\square$ (Right) around $x \sim 0.92$. This means that the deviation $\Delta x$ is $\lesssim 2 \sigma$ for $N \leq 16$. Thus, the remaining overlap problem associated with this observable is practically small. This is consistent with the fact that we were able to reproduce the GEM result by constraining only the four observables $\lambda_{n}(n=1,2,3,4)$.

\section{Conclusions}

In this work, we have applied the factorization method to the Monte Carlo study of a matrix model with strong complex action problem. It has been extended to include more than one observables in order to eliminate the overlap problem and sample effectively the theory's configuration space. A maximal set $\Sigma$ of such observables has been determined that has significant correlations with the complex phase. By constraining sampling to configurations that lie in the neighbourhood of the solution to the minimization of the free energy of the distribution functions of the observables in $\Sigma$, all other observables can be computed without the complex action problem. The complex action problem has been reduced to solving the saddle point equations, which is difficult, but not 
impossible if one takes advantage of the nice scaling properties of the factors in the distribution functions. Solving the equations for the stationary configurations of $\Gamma$ played an important role in determining $\Sigma$.

Our results turn out to be consistent with analytical calculations using the GEM and confirm SSB of rotational invariance in the distribution of eigenvalues of bosonic matrices. This is related to the problem of dynamical compactification of extra dimensions in string theory which will be studied further in the IIB matrix model as well as in simpler matrix models with supersymmetry.

The steps followed in this study are considered to be generic and we hope that a similar approach can be applied to many interesting systems.

\section{References}

[1] K.N. Anagnostopoulos, J. Nishimura, Phys. Rev. D 65, 105012 (2002).

[2] J. Ambjørn, K. N. Anagnostopoulos, J. Nishimura and J. J. M. Verbaarschot, JHEP 0210 (2002) 062; Phys. Rev. D 70 (2004) 035010; V. Azcoiti, G. Di Carlo, A. Galante and V. Laliena, Phys. Rev. Lett. 89 (2002) 141601; Z. Fodor, S. D. Katz and C. Schmidt, JHEP 0703 (2007) 121; S. Ejiri, Phys. Rev. D 77, 014508 (2008); K. N. Anagnostopoulos, T. Azuma, J. Nishimura, PoS LATTICE2010, 167 (2010).

[3] K.N. Anagnostopoulos, T. Azuma, J. Nishimura, Phys. Rev. D83, 054504 (2011); J. High Energy Phys. 10 (2011) 126.

[4] W. Krauth, H. Nicolai and M. Staudacher, Phys. Lett. B 431, 31 (1998); T. Hotta, J. Nishimura and A. Tsuchiya, Nucl. Phys. B 545, 543 (1999); J. Ambjørn et. al., JHEP 0007, 013 (2000); JHEP 0007, 011 (2000); Phys. Rev. D 65, 086001 (2002); P. Bialas et. al.,Nucl. Phys. B 592, 391 (2001); Z. Burda et. al., Nucl. Phys. B 602, 399 (2001); JHEP 0503, 058 (2005); S. -W. Kim, J. Nishimura,

A. Tsuchiya, arXiv:1108.1540 [hep-th]; arXiv:1110.4803 [hep-th].

[5] O. Aharony et. al., Class. Quant. Grav. 21 (2004) 5169; JHEP 0601, 140 (2006); M. Hanada, J. Nishimura and S. Takeuchi, Phys. Rev. Lett. 99, 161602 (2007); S. Catterall and T. Wiseman, JHEP 0712, 104 (2007); K. N. Anagnostopoulos, M. Hanada, J. Nishimura and S. Takeuchi, Phys. Rev. Lett. 100, 021601 (2008); S. Catterall and T. Wiseman, Phys. Rev. D 78, 041502 (2008); S. Catterall and T. Wiseman, JHEP 1004 (2010) 077; M. Hanada et. al., Phys. Rev. Lett. 102, 191602 (2009); Phys. Rev. Lett. 102, 181602 (2009); Phys. Rev. Lett. 104, 151601 (2010); S. Catterall, A. Joseph and T. Wiseman, arXiv:1008.4964 [hep-th].

[6] N. Ishibashi, H. Kawai, Y. Kitazawa and A. Tsuchiya, Nucl. Phys. B 498, 467 (1997); H. Aoki, S. Iso, H. Kawai, Y. Kitazawa and T. Tada, Prog. Theor. Phys. 99 (1998) 713; for a review see e.g. H. Aoki et. al., Prog. Theor. Phys. Suppl. 134 (1999) 47; T. Azuma, arXiv:hep-th/0401120.

[7] J. Nishimura and F. Sugino, JHEP 0205 (2002) 001; H. Kawai et. al., Nucl. Phys. B 647, 153 (2002); Prog. Theor. Phys. 109, 115 (2003); T. Aoyama, J. Nishimura, T. Okubo, Prog. Theor. Phys. 125, 537-563 (2011); J. Nishimura, T. Okubo, F. Sugino, arXiv:1108.1293 [hep-th].

[8] J. Nishimura, Phys. Rev. D 65, 105012 (2002).

[9] J. Nishimura, T. Okubo and F. Sugino, Prog. Theor. Phys. 114, 487 (2005).

[10] J. Nishimura, G. Vernizzi, JHEP 0004, 015 (2000); Phys. Rev. Lett. 85, 4664-4667 (2000). 